\title{
HealthSense: An Application for Querying Raw Sensor Data ${ }^{\star}$
}

\author{
Fabrice Camous, Dónall McCann, and Mark Roantree \\ Interoperable Systems Group, \\ School of Computing, Dublin City University, \\ Dublin, Ireland \\ \{fcamous, dmccann, mroantree\}@computing.dcu.ie
}

\section{Background}

New sensing technologies and the decreasing cost of Information and Communication Technologies (ICTs) make possible the development of electronic Health (eHealth) monitoring systems. The challenges of such systems include the representation of data extracted from various sensor devices by knowledge workers through semantic enrichment and integration. Also, the data must be stored in a format suitable for querying and further analysis. This paper describes the demonstration of the HealthSense system which captures and queries personal health data extracted from wearable sensors. Figure 1 illustrates the transformation process. There are 4 layers, representing data in different formats, separated by the 3 processors that transform them. A detailed description of the 3 processors was presented in [1].

\section{The HealthSense Demonstration}

The demonstration includes:

- the wearing of sensor devices and recording of data,

- the extraction of sensor data to a laptop,

- the use of HealthSense to enrich, integrate, and store sensor data, and

- the querying of the stored data from an XML database using XPath.

\subsection{The Sensor Devices}

- Polar S625X ${ }^{\mathrm{TM}}$ heart-rate monitor: this consists of a fabric band which fits around a person's chest and detects and logs their heart rate.

- BodyMedia SenseWear@: this sensor array is worn around the upper arm and measures. It uses motion sensors and galvanic skin response sensors to measure activity.

- Deluxe Wrist Blood Pressure Monitor HL168JC: this device can store up to 90 blood pressure and pulse readings.

- iPod Nano 4G with Nike®+ IPod Sport kit: this sensor records the distance covered during a walk or run and caloric consumption. 


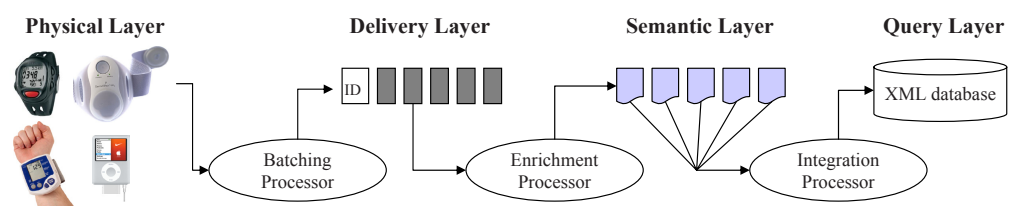

Fig. 1. System Architecture

\subsection{Data Extraction, Enrichment and Integration}

Device-specific software is used to extract and store the raw sensor data on the laptop hard disk. At this point, data is in a raw format not yet queryable in a standard way. The HealthSense application running on the laptop is used to batch, semantically enrich, integrate, and store the data locally in a XML database. Processing time depends on the size of the files. Table 1 shows examples of processing times from file upload to database storage for different groups of files. A complete analysis of processing times for enrichment and integration is found in [1]. For storage, we use the eXist XML database [2]. Our innovation arises from the fact that we can enrich data from any sensor device using our generic device manager, and our integration process can pivot on a number of different characteristics including time, individuals or sensor readings [1]. Once the data is stored in eXist, it is directly queryable using the XPath query language.

Table 1. Processing times from file upload to database storage

\begin{tabular}{|l|l|l|}
\hline Raw Sensor Files & $\begin{array}{l}\text { Upload to } \\
\text { Storage (sec) }\end{array}$ & $\begin{array}{l}\text { Stored File } \\
\text { Size }\end{array}$ \\
\hline 1 Polar (3KB), 1 BodyMedia (173KB) & 3.90841 & $388 \mathrm{~KB}$ \\
\hline 1 Nike iPod (8KB), 1 Polar (13KB), 1 BodyMedia (437KB) & 7.31041 & $1060 \mathrm{~KB}$ \\
\hline 1 Nike iPod (8KB), 2 Polar (23KB), 1 BodyMedia (1099KB) & 12.22107 & $2424 \mathrm{~KB}$ \\
\hline
\end{tabular}

\section{References}

1. Camous, F., McCann, D., Roantree, M.: Capturing Personal Health Data From Wearable Sensors. In: Proc. of the 2nd Intl. Workshop on SensorWebs, Databases and Mining in Networked Sensing Systems (to appear, 2008)

2. Meier, W.: Index-Driven XQuery Processing in the eXist XML Database. In: XML Prague 2006 (2006) 